\title{
Arguments For and Against Limited Relativistic Transformations for Any Signal Velocity
}

\author{
Mario Rabinowitz \\ Armor Research, 715 Lakemead Way, Redwood City, CA 94062-3922 USA \\ Email: mario715@gmail.com
}

\begin{abstract}
Time dilation derived here from relativistic Least Action couples relativistic mass and time transformations. This is an argument against time dilation for other than the signal speed of light $c$. Because $c$ is the paramount signal velocity in Special Relativity, we examine the extent to which limited relativistic transformations of time dilation and length contraction might occur for any signal velocity, $C<c$, provided that the relative frame velocity $v \ll<C$. Such relativistic effects depending on $C$ may be detectable in a narrow range. If allowed by nature and confirmed by experiment, this will alter our views of space-time. Known experiments do not rule this out. The total resultant velocity $v_{3}$ cannot exceed $C$ in the Einstein addition of velocities due to the condition $v \ll<C$. A derivation is presented which together with experiment rules out mass transformation unless the signal velocity is the speed of light.
\end{abstract}

Keywords: Limited time and length transformations, relativistic least action, universal relativistic effects, time dilation, length contraction, relativistic mass increase, generalized signal velocity, special relativity.

\section{Introduction}

In addition to two explicit postulates, two implicit postulates in Einstein's Special Relativity (ESR) paper of 1905 [1] are that the geometry is Euclidean, and that $c$ is the signal speed of light (though light signals are mentioned). The present understanding is that no "signal or new information" can be communicated faster than the signal speed of light in vacuum $c=299,792,458 \mathrm{~m} / \mathrm{sec}$. [Group velocities can exceed $c$, or be $<10^{3} \mathrm{~m} / \mathrm{s}$ by changing the central frequency of a light pulse.] This paper examines the foundational role of $c$ in the kinematic part of relativity theory, and finds that in a limited range of frame velocity a signal velocity $C<c$ may give quasi-relativistic effects for time dilation and length contraction. Theoretical analysis in conjunction with experiment rules out relativistic mass increase unless the signal velocity is the speed of light. A derivation is presented of time dilation and length contraction for a signal velocity $C$ that, within the approximation made, follows Einstein's derivation. It is shown that if universal relativistic effects for time and length as a function of $C$ exist, they may be detectable in a narrow range. Since this has not been previously considered, this paper will examine the pros and cons. For a balanced perspective, the Relativistic Principle of Least Action is next used for a novel derivation of time dilation thus linking it to mass increase.

\section{Relativistic Least Action Principle Couples Mass and Time Transformations}

The action between the times $t_{1}$ and $t_{2}$ is

$$
S\left(t_{1}, t_{2}\right) \equiv \int_{t_{1}}^{t_{2}} \Lambda(r, v, t) d t
$$

The function $\Lambda$ is the Lagrangian $\Lambda \equiv \mathrm{K}-V$, where $\mathrm{K}$ is the kinetic energy of the particle, and $V$ is its potential energy. Let us choose $V=0$ and one-directional motion of an uncharged, free particle of rest mass $m_{0}$ with velocity $v$ at time $t$, in the context of Einstein's Special Relativity (ESR). [Non- 
relativistically $\mathrm{K}=\frac{1}{2} m_{0} v^{2}$.] The action $S$ is a functional which allocates a number to each possible path such that this number is an extremum (minimum, maximum, or stationary) for that path which results from the laws of motion. [It is referred to as the principle of least action because the extremum is usually a minimum.] The Least Action Principle is a powerful tool since it can be used to solve both classical and quantum mechanical systems that cannot be solved by Newton's equations nor directly by a Hamiltonian. In such systems, the force on a mass at a given time depends on the motion of the mass at other times, especially when retarded (and if advanced) potentials are at play. $\mathrm{S}$ is invariant (frame independent) because The Laws of Physics are Frame Independent is the second explicit postulate of ESR [1].

The relativistic momentum $p=\frac{m_{0} v}{\sqrt{1-v^{2} / c^{2}}}=\frac{\partial \Lambda}{\partial v}$. So for $V=0$, this

$$
\Rightarrow \Lambda=m_{0} \int \frac{v}{\sqrt{1-v^{2} / c^{2}}} d v=-m_{0} c^{2} \sqrt{1-v^{2} / c^{2}}
$$

This may be surprising since the relativistic kinetic energy is $\mathrm{K}=m_{0} c^{2} \sqrt{1-v^{2} / c^{2}}-m_{0} c^{2}$ so that one might expect $\Lambda \equiv \mathrm{K}-V=\mathrm{K}-0=m_{0} c^{2} \sqrt{1-v^{2} / c^{2}}-m_{0} c^{2}$. This was sorted out after 1942. [Both forms reduce to $\mathrm{K}=\frac{1}{2} m_{0} v^{2}$ non-relativistically.] Eqs. (1) and (2)

$$
\Rightarrow S\left(t_{1}, t_{2}\right)=-m_{0} c^{2} \sqrt{1-v^{2} / c^{2}} \int_{t_{1}}^{t_{2}} d t=-m_{0} c^{2} \sqrt{1-v^{2} / c^{2}}\left(t_{2}-t_{1}\right)
$$

The action $S\left(t_{1}, t_{2}\right)$ is frame independent if the time interval $t_{2}-t_{1}$ in the moving frame is proportional to the time interval in the rest frame $t_{02}-t_{01}$ thus:

$$
t_{2}-t_{1}=\frac{t_{02}-t_{01}}{\sqrt{1-v^{2} / c^{2}}}
$$

It appears this is the first time that the relativistic mass and time transformations have been linked, thus relating a dynamic and a kinematic transformation. The mass variation analysis in Sec. 5 makes it look unlikely that $C$ can replace $c$ in Eq. (4) because Eq. (4) results from $m(v, c)$. Furthermore since Einstein [1] showed that the time and length transformations are reciprocal coordinate transformations this also argues against $C$ replacing $c$ in the length transformation. Nevertheless $T(v, C)$ and $L(v, C)$ are derived directly and independently of $m(v)$ in Sec. 3. This issue should be decided experimentally which is the final authority in physics.

\section{$3 \quad$ Time Dilation and Length Contraction for Any Signal Speed}

Following Einstein's derivation [1] approximately, let us consider an almost spherical wave of signal velocity $C \neq c$ between two frames with relative velocity $v$ in the $x$ direction. In this idealized thought experiment, at time $t=0$ a point like signal source fixed at the origin of each system emits a signal pulse of velocity $C$ when their origins coincide.

To achieve the approximation of a spherical wave we require $C \pm v \approx C$, i.e. $v<<<$. This is the approximate equivalent of Einstein's explicit first postulate that $c$ is constant with respect to all frames. There is a spherical wave front in the stationary unprimed system

$$
x^{2}+y^{2}+z^{2}=C^{2} t^{2}
$$

There is an approximately spherical wave front in the primed system moving uniformly with velocity $v$,

$$
x^{\prime 2}+y^{\prime 2}+z^{\prime 2}=(C \pm v)^{2} t^{\prime 2} \approx C^{2} t^{\prime 2}
$$

Here the step is taken that not only space but time transforms from one system to another.

Equating Eqs. (5) and (6) for the two coordinate system wave fronts:

$$
x^{2}+y^{2}+z^{2}-C^{2} t^{2} \approx x^{\prime 2}+y^{\prime 2}+z^{\prime 2}-C^{2} t^{\prime 2}
$$


Following Einstein's strategy, with Eq. (7) as a starting point, the corresponding approximate equations for length contraction and time dilation can be derived with the speed of light $c$ replaced by the signal velocity $C$. These derivations can be found in relativity textbooks. Rest frames will be designated by either a subscript 0 or no subscript, and moving frames by a prime '. Subscripts 1 and 2 designate different positions $\chi$ and times $t$.

$$
\begin{gathered}
x_{1} \approx \frac{x_{1}{ }^{\prime}-v t}{\sqrt{1-v^{2} / C^{2}}} \text { and } x_{2} \approx \frac{x_{2}{ }^{\prime}-v t}{\sqrt{1-v^{2} / C^{2}}} \\
\Rightarrow L_{0} \equiv x_{2}-x_{1} \approx \frac{x_{2}{ }^{\prime}-x_{1}{ }^{\prime}}{\sqrt{1-v^{2} / C^{2}}} \equiv \frac{L^{\prime}}{\sqrt{1-v^{2} / C^{2}}} \\
\Rightarrow L^{\prime} \approx L_{0} \sqrt{1-v^{2} / C^{2}} \text { for } v<<<C \\
t_{1}{ }^{\prime} \approx \frac{t_{1}-v x_{1} / C^{2}}{\sqrt{1-v^{2} / C^{2}}} \text { and } t_{2}{ }^{\prime} \approx \frac{t_{2}-v x_{1} / C^{2}}{\sqrt{1-v^{2} / C^{2}}} \\
\Rightarrow T^{\prime} \equiv t_{2}{ }^{\prime}-t_{1}{ }^{\prime} \approx \frac{T_{0}}{\sqrt{1-v^{2} / C^{2}}} \text { for } v<<<C
\end{gathered}
$$

\section{Domain for Length and Time Effects for Any Signal Speed}

Let us determine a domain of possible validity by ascertaining how large an effect can be expected for the condition $v<<<$ when $(v / C) \sim 10^{-3}$ for any $C$. As an extreme example let's use sound for the signal velocity $C$. Its speed in air is $\approx 340 \mathrm{~m} / \mathrm{sec}$. $(\approx 1000 \mathrm{ft} / \mathrm{sec})$, so a velocity $v \leqslant 0.3 \mathrm{~m} / \mathrm{sec}$ $(\approx 1 \mathrm{ft} / \mathrm{sec})$ would do for the moving frame. [ $\left.C_{\text {water }}=1484 \mathrm{~m} / \mathrm{sec} . C_{\text {iron }}=5120 \mathrm{~m} / \mathrm{sec}\right]$.

For length contraction $L^{\prime} \approx L_{0} \sqrt{1-v^{2} / C^{2}}$ as given by Eq. (10), we have

$$
\frac{L^{\prime}}{L_{0}} \approx\left[1-(v / C)^{2}\right]^{1 / 2}=\left[1-\left(10^{-3}\right)^{2}\right]^{1 / 2}=\left[1-10^{-6}\right]^{1 / 2} \approx 1-10^{-6} / 2
$$

For $(v / C) \sim 10^{-3}$, a length will be reduced by $\sim 10^{-6} / 2$. So a $200 \mathrm{~m}$ long rod, would be expected to shrink by $10^{-4} \mathrm{~m}=0.1 \mathrm{~mm}$ when its speed is $\approx 0.3 \mathrm{~m} / \mathrm{sec}$. For the detection of gravitational waves, length is incredibly measured to $\sim 10^{-15} \mathrm{~m}$, within the diameter of a proton. Our required observation is $10^{11}$ longer. So this measurement should be readily possible. A $\geq 200$ meter rod would not have to be moved, but just the detector since only the relative velocity is relevant. To avoid unlikely but possible complications due to rotation and revolution of the earth, the rod should be oriented approximately parallel to the longitude i.e. perpendicular to the motion of the earth.

For time dilation as given by Eq. (12), for a frame moving at $\approx 0.3 \mathrm{~m} / \mathrm{sec}$ we have

$$
\frac{T^{\prime}}{T_{0}} \approx\left[1-(v / C)^{2}\right]^{-1 / 2}=\left[1-\left(10^{-3}\right)^{2}\right]^{-1 / 2} \approx 1+10^{-6} / 2
$$

So a rest-frame time will be increased (dilated) by $\sim 10^{-6} / 2$ by using sound as a signal velocity. A sound clock could beep recorded signals; or by ultrasound imaging where an acoustic wave could make a sonar image of a clock in the observer's frame as the observer rides toward the acoustic beam. The clock appears to run slower and slower as the speed of sound is approached. At the speed of sound the clock would appear to stop. When the speed of sound is exceeded, the clock would appear to run backward. This is somewhat like Einstein's thought experiment of catching up with a moving light beam while observing a stationary clock on top of city hall.

Atomic clocks are the most accurate clocks known. They use an electronic transition frequency in the microwave, optical, or ultraviolet part of the atomic spectrum for time keeping, and are used in GPS (global position satellites). They have an accuracy of $10^{-9} \mathrm{sec} /$ day(day/86400 sec) $\sim 10^{-14}$. The $10^{-6}$ time dilation effect is $10^{8}$ greater than this, so it may be possible to use atomic clocks to ascertain time dilation for a less accurate sonar clock. 
As a small part of a paper in January 2016, Rabinowitz introduced the concept that limited relativistic time $\mathrm{T}$ and length $\mathrm{L}$ may be observable for signal velocities other than the velocity of light.[2] The present paper goes into greater depth as well as considering questions not covered in that paper, such as the question of mass.

\section{Only the Speed of Light Is Appropriate for Mass Increase Transformation}

We want to test the assumption that for relativistic mass increase in a small domain the speed of light $c$ can be replaced by a signal velocity $C \gg>v$, the body's velocity. The relation between energy and work done $d E=F \cdot d x=F \cdot v d t$ or equivalently power $P=d E / d t=F \cdot v$ is fundamental, with the force, $F=d(m v) / d t$. It is the starting point to ascertain if this assumption contradicts physical observation. We will attempt to determine if the signal velocity $C$ can differ significantly from the speed of light.

We assume the energy $E=f m(C \pm v)^{2} \approx f m C^{2}$, where $0 \leq f \leq 1$; and $(C \pm v)^{2} \approx C^{2}$ is the inexact equivalent of Einstein's postulate [1] that $c$ is constant with respect to all frames.

$$
\frac{d E}{d t}=\frac{d\left[f m(C \pm v)^{2}\right]}{d t} \approx \frac{d\left[f m C^{2}\right]}{d t}=F \cdot v=\frac{d}{d t}\left[\left(\frac{d(m v)}{d t}\right) \cdot v\right]=\frac{d}{d t}\left[m \frac{d v}{d t}+v \frac{d m}{d t}\right] v
$$

where $m$ is the mass of the moving body, and $f=1$ for $C=c$. From equation (15):

$$
\begin{aligned}
& f C^{2} \frac{d m}{d t}=m v \frac{d v}{d t}+v^{2} \frac{d m}{d t} \rightarrow \int_{m_{0}}^{m} \frac{d m}{m}=\int_{v_{0}}^{v} \frac{v d v}{\left(f C^{2}-v^{2}\right)} \rightarrow \ln \left(\frac{m}{m_{0}}\right) \\
& =\frac{-1}{2}\left\{\ln \left[1-\frac{v^{2}}{f C^{2}}\right]\right\}
\end{aligned}
$$

With $v_{0}=0 \Rightarrow m_{0}$ is the rest mass in the rest frame. So Eq. (16) $\Rightarrow$

$$
m=\frac{m_{0}}{\sqrt{1-v^{2} / f C^{2}}}
$$

Notably in this derivation: The velocity $v$ can be time varying, since there was no restriction that the velocity $v$ be constant. Since no Time nor Length transformations were invoked, this Mass transformation is independent of them.

In Eq. (17) with $C=c$, multiplying by $c^{2}$ let us see if we can determine $f$.

$$
E=f m c^{2}=f m_{0} c^{2}\left[1-\frac{v^{2}}{f c^{2}}\right]^{-1 / 2}
$$

For $v<<c$, a binomial expansion of Eq. (18) yields the well-known result:

$$
E=f m_{0} c^{2}\left[1+\frac{1}{2} \frac{v^{2}}{f c^{2}}+\frac{3}{8} \frac{v^{4}}{f^{2} c^{4}}+\ldots\right] \approx f m_{0} c^{2}+\frac{1}{2} m_{0} v^{2}
$$

Therefore with $f=1, E=m c^{2}$ is consistent and isomorphic with physics data. Note that the low velocity equation for kinetic energy, $K E=\frac{1}{2} m_{0} v^{2}$ is unchanged regardless of the value of $f$.

It has been shown that if we assume an equation of the form $E=m c^{2}$, by the conservation of energy it follows that an equation of the form $m=m_{0} / \sqrt{1-v^{2} / c^{2}}$ must also be obeyed. Similarly, by the conservation of energy, it follows that if we assume $E=f m C^{2}$, then $m=m_{0} / \sqrt{1-v^{2} / f C^{2}}$ must also be obeyed. Since $E=m c^{2}$ has been measured with high accuracy, the analysis is valuable in determining the domain where $m=m_{0} / \sqrt{1-v^{2} / f C^{2}}$ with $f=1$ is or is not compatible with physical data.

In 2005, Rainville et al. [3] conducted a direct test of the energy-equivalence of mass lost in the binding energy of a neutron to atoms of particular isotopes of silicon and sulfur. They compared the 
mass lost to the energy of the emitted gamma ray associated with the neutron capture. The binding mass-loss agreed with the gamma ray energy to a precision of $0.00004 \%$, the most accurate test of $E=m c^{2}$ to date. Although they said $E=m c^{2}$, they implicitly meant $E=\Delta m c^{2}$ where $\Delta m$ is the change in mass.

The analysis shows that a test of the accuracy of $E=m c^{2}$ subsumes testing the accuracy of mass increase with velocity as given by Eq. (17). The Rainville et al. accuracy

$$
\begin{aligned}
& \Rightarrow\left(\frac{C}{c}\right)^{2} \approx 1-4 \times 10^{-7} \rightarrow C \approx\left(1-4 \times 10^{-7}\right)^{1 / 2} c \approx\left(1-2 \times 10^{-7}\right) c \approx c \\
& =3 \times 10^{8} \mathrm{~m} / \mathrm{sec}
\end{aligned}
$$

Since experiment is the final arbiter, the highly accurate Rainville et al. experiment in conjunction with Eq. (17) implies that only the speed of light can be used for mass increase. This rules out any other signal velocity within an error of $\pm 2 \times 10^{-7} c$.

Unlike the proofs in Sec. 2, the proof in this section uncouples the mass $\mathrm{m}$ from $\mathrm{T}$ and $\mathrm{L}$; and even allows the mass to have any time varying velocity. The $\mathrm{T}$ and $\mathrm{L}$ transformations are kinematic, i.e the motion of objects without reference to forces. The $m$ transformation is dynamic, i.e. the motion of bodies under the action of forces. Even for theoretical coupling (cf. Sec. 2) of m, and T or L transformations, experiments should still be done to ascertain if quasi-relativistic kinematic effects exist for a signal velocity $C<c$.

Some argue that relativistic mass should be abandoned because the increase of mass with velocity originates not in the object but in the geometric properties of space-time itself. Einstein abandoned the concept of velocity dependent mass, saying in 1948 that it is not good to introduce the concept of the [relativistic] mass of a moving body for which there is no clear definition. Despite his 1948 comment, many distinguished relativity experts such as M. Born, V. Fock, F. Klein, M. Laue, and R. Tolman in the past; and W. Rindler, and R. Penrose in recent times use the concept of relativistic mass.

The concept of relativistic mass comes about naturally from electromagnetic mass in which c enters intrinsically. For those who think mass as a function of velocity is electromagnetic in origin, the Poynting vector $\mathbf{S}=\mathbf{E} \times \mathbf{H}$ can be used in Eq. (15).

$$
\frac{d\left(m c^{2}\right)}{d t}=\int \mathbf{E} \times \mathbf{H} d A=F \cdot v=\frac{d}{d t}\left[\left(\frac{d(m v)}{d t}\right) \cdot v\right]=\frac{d}{d t}\left[m v \frac{d v}{d t}+v^{2} \frac{d m}{d t}\right]
$$

where $\mathbf{E}$ is the electric field vector, and $\mathbf{H}$ is the magnetic field vector as related by Maxwell's equations; and $A$ is area.

\section{Discussion}

If one transforms from Frame 1 (system 1) to Frame 2 with a relative velocity $v_{1}$, and then Frame 3 with a relative velocity $v_{2}$, the total velocity $v_{3}$ is given by the Einstein addition of velocities:

$$
v_{3}=\frac{v_{1}+v_{2}}{1+v_{1} v_{2} / c^{2}}
$$

Such transformations don't exceed the speed of light $c=299,792,458 \mathrm{~m} / \mathrm{sec}$ in vacuum.

$$
\text { For } v<<<C: v_{3} \approx \frac{v_{1}+v_{2}}{1+v_{1} v_{2} / C^{2}}
$$

In Eq. (23), let $v_{1} \equiv g C, v_{2} \equiv h C$ with $g<<1$, and $h \ll<1 \Rightarrow$

$$
v_{3} \approx \frac{g C+h C}{1+g h C^{2} / C^{2}}=\frac{(g+h) C}{1+g h}<<C \leq c
$$

Equation (24) shows that the reduced signal speed $C$ cannot be exceeded for any $g<<1$ and $h<<1$; i.e. any $v_{1}<C$ and $v_{2}<C$. 
The signal velocity $v_{s}$, group velocity $v_{g}$, and energy transfer velocity are all the same, $c=3 \times 10^{8} \mathrm{~m} / \mathrm{sec}$ for non-absorbing media such as vacuum, but can differ in an absorption band. In the past, experimental $v_{g}>c$ was considered in conflict with ESR. Sommerfeld resolved this by showing that $v_{s} \leq c$ even for anomalous dispersion where $v_{g}>c$. In an absorptive medium, $v_{s}<c$ for ordinary detectors. [4] This is only relevant if $v_{s}$ is not constant with respect to absorptive media. Also very sensitive detectors may find $v_{s} \approx c$. [4] No properties of light were invoked in Einstein's derivation [1] of relativistic length and time, other than postulating that $\mathrm{c}$ is constant with respect to all frames. That $\mathrm{c}$ is constant with respect to source frames is true anyway for wave propagation in a medium. To my knowledge, c constant with respect to observer frames and Einstein's addition of velocities have not been well verified experimentally.

Einstein showed that the kinematic time and length transformations are reciprocal coordinate transformations where the equations have the same structure with the interchange of time $t$ and length $x$ due to their symmetrical form in eqs. (8) and (11). So one might expect time contraction to be similar to length contraction. Yet one gets time dilation.

One of Einstein's unique contributions [1] was the abandonment of the ether in his postulate that $c$ is constant relative to all observers. He stated [5] that he was well aware of the Lorentz transformation and the Michelson-Morley experiments of 1881 and 1887. He said he heard of these experiments in his student days when he conceived of a similar but much less sensitive experiment using thermocouples to measure the temperature difference between light beams reflected parallel and anti-parallel to the earth's motion. [5] In fact in [1] he says "the unsuccessful attempts to discover any motion of the earth relatively to the 'light medium,' suggest that the phenomena of electrodynamics as well as of mechanics possess no properties corresponding to the idea of absolute rest."

For the condition $v<<<$, Eqs. (10) and (12) are almost Galilean transformations, which they would be if $(v / C) \rightarrow 0$. However within the realm of possible experimental measurement, they remain Lorentz transformations because even though $(v / C)$ is very small, it is not 0. Eqs. (10) and (12) were derived prior to Einstein by Lorentz, assuming an absolute rest frame (ether) for velocities, and named the Lorentz Transformation by Poincare in 1905. Lorentz conjectured that the speed of light [in vacuum] might be a limiting speed.[6] It is clear that Lorentz thought long about it and showed an early concern. In a letter (August 1892) to Lord Rayleigh, Lorentz wrote: "Can there be some point in the theory of Mr. Michelson's experiment [interferometer null results to measure the motion of the earth through a presumed ether] which has yet been overlooked?"

In 1899 Lorentz also wrote a paper in English, Simplified Theory of Electrical and Optical Phenomena in Moving Systems.[7] In it, he wrote, "The ions were supposed to be perfectly permeable to the ether, so that they can move while the ether remains at rest." So the reader can move more comfortably from his book to the excellent English in this paper, Lorentz starts from the same hypotheses and introduces the same notations. He goes on to say, "If the hypothesis might be taken for granted, Michelson's experiment should always give a negative result, whatever transparent media were placed on the path of the rays of light, and even if one of these went through air, and the other, say through glass."

It is clear that Lorentz had two kinds of time in mind-universal time and local time-. "The last of these is the time, reckoned from an instant that is not the same for all points of space, but depends on the place we wish to consider. We may call it the local time, to distinguish it from the universal time t." [7] Whereas Einstein [1] more generally distinguished between time in the rest frame and in the moving frame. [8]

Joseph Larmor [9] appears to have anticipated time dilation in 1897 prior to Lorentz and Einstein in saying "individual electrons describe corresponding parts of their orbits in times shorter for the [rest] system in the ratio $\sqrt{1-v^{2} / c^{2}}$." Yet as late as 1927 (22 years after Einstein Special Relativity) he argued that an absolute time was crucial to astronomy.

Despite the priority of Lorentz and others for length $\mathrm{L}(v)$, time $\mathrm{T}(v)$, and mass $m(v)$ transformations, Einstein justly deserves credit for the greater, deeper, richer, and more unified development of Special Relativity as presented in his 1905 paper [1,2,8]. Einstein's $\mathrm{L}(v)$ and $\mathrm{T}(v)$ transformations are kinematic and precise, whereas Lorentz' are dynamic and approximate. Einstein merits total credit for 
priority in his monumental conception and development of the theory of General Relativity. However, the popular acclaim given to him for the equation relating energy and mass, $E=m c^{2}$, is unjustified by historical record. [2.8] In Einstein's first 1905 paper he obtained an inappropriate equation for $m(v)$ which is related to $E=m c^{2}$.[1] In 1904 Lorentz derived $m(v)=m_{0} /\left[1-\left(v^{2} / c^{2}\right)\right]^{1 / 2}$ for the special case of a deformable electron[10]. It is commonly thought that Einstein derived $E=m c^{2}$ for any mass (not just electromagnetic mass) in his 2nd 1905 paper.[11] However, he only suggested non-electromagnetic mass in saying: "The fact that the energy withdrawn from the body becomes energy of radiation evidently makes no difference...." His proof of radiation recoil [11] is preceded by many electromagnetic $E=m c^{2}$ proofs including Hasenöhrl's photon in a box where evidently the electromagnetic mass could also be interpreted more generally.[2, 12] Even if credit for $E=m c^{2}$ is taken away, Einstein still remains one of the greatest physicists that ever lived.

\section{Conclusion}

By using a signal velocity $C$ different than that of light $c$, and following reasoning similar to that of Einstein's 1905 paper [1], derivations herein showed that relativistic time dilation $\mathrm{T}$ and length contraction L may be possible in limited domains. Known experiments do not rule this out. As shown in the present paper, relativistic mass increase is ruled out. Experiment will decide the issue about $\mathrm{T}$ and $\mathrm{L}$. In Sec. $5, \mathrm{~m}(v)$ was derived completely independent of $\mathrm{L}(v)$ and $\mathrm{T}(v)$; and $\mathrm{T}(v, C)$ was derived directly and independently of $\mathrm{m}(v)$ in Sec. 3. Yet, $\mathrm{T}(v)$ was derived from the Relativistic Least Action Principle in Sec.2, showing that $\mathrm{T}(v)$ and $\mathrm{m}(v)$ can be coupled. Thus the validity of using $C$ may need to be decided experimentally. Even if the relativistic transformation related to $(v / c)^{2}$ is negligible, $(v / C)^{2}$ may not be negligible because it is $(c / C)^{2}$ times larger. If experiment decides that limited relativistic effects are possible using $C$, this will be an exciting revelation that will give new insights and open up new vistas with respect to our views of space-time. If it turns out that one can only use $c$, this will further point to the importance of light, electromagnetic phenomena, and space-time. Hopefully this paper will serve as an incentive to investigate this issue.

\section{References}

1. A. Einstein, "On the electrodynamics of moving bodies," Annalen der Physik, vol. 17, pp. 891- 921, 1905.

2. M. Rabinowitz, "Thoroughly testing Einstein's special relativity theory, and more," Journal of Modern Physics, vol. 7, pp. 87-105, 2016. http://dx.doi.org/10.4236/jmp.2016.71009

3. S. Rainville et al, "A direct test of $\mathrm{E}=\mathrm{mc} 2$," Nature, vol. 438, pp. 1096 - 1097, 2005.

4. J. D. Jackson, Classical Electrodynamics, p.319, Wiley, 1975.

5. A. Einstein, "How I created the theory of relativity," Physics Today vol. 35 no. 8 pp. 45-47,1982.

6. H. A. Lorentz. in Vereenvoudigde Theorie der Electrische en Optische Verschijnselen in Lichamen, die Zich Bewegten Korpern, Koninklijke Akad. Wetenschappen, 1899.

7. H. A. Lorentz, "Simplified theory of electrical and optical phenomena in moving systems," Proc. Royal Netherlands Acad. of Arts and Sciences, vol. 1, pp. 427, 1899.

8. M. Rabinowitz, "General derivation of mass-energy relation without electrodynamics or Einstein's postulates," $J$. Mod. Phys. vol. 6, pp.1243-1248, 2015. http://dx.doi.org/10.4236/jmp.2015.69129

9. J. Larmor, "A dynamical theory of the electric and luminiferous medium Part III," Phil. Trans. Roy. Soc. 190, pp. 205-289, 1897.

10. H. A. Lorentz, "Electromagnetic phenomena in a system moving with any velocity smaller than that of light," Proc. Royal Netherlands Acad. of Arts and Sciences, vol.6, pp. 809-831, 1904.

11. A. Einstein, "Does the inertia of a body depend upon its energy-content," Annalen der Physik, vol. 18, pp. 639641, 1905.

12. F. Hasenöhrl, "On the theory of radiation between bodies," Annalen der Physik, vol. 320, pp. 344-370 1904. 